\title{
Ensayo Pictórico: Control imagenológico de aneurismas tratados ¿Qué examen elegir?
}

Mario Zerega Ruíz', Karin Müller Campos', Isidro Huete Lira', Juan Pablo Cruz Quiroga', Sebastián Bravo Grau'.

1.Departamento de Radiología, Unidad de Neurorradiología, Facultad de Medicina. Hospital Clínico de la Pontificia Universidad Católica de Chile. Santiago, Chile.

\section{Pictorial Essay: Imaging control of treated aneurysms. Which exam to choose?}

\begin{abstract}
Treatment of intracranial ruptured and unruptured aneurysms can be performed with surgical (vascular clips) or endovascular procedures (coils, stents, flow diverters). There are several devices and techniques for endovascular treatment. Knowledge of this techniques allows physicians to choose and interpret accurately imaging modalities for non-invasive follow-up.

Through a pictorial essay of imaging follow-up of treated intracranial aneurysms we show the differences in characterization with computed tomography angiography, magnetic resonance angiography without contrast (time of flight or "TOF" technique), contrast enhanced MR angiography $\left(C_{+}\right)$and digital subtraction angiography (DSA). Additionally we recommend imaging modalities for each treatment device.

Keywords: brain aneuryms, coils, intracranial stent, flow diverter, vascular clips, imaging follow-up.
\end{abstract}

Resumen. El tratamiento de aneurismas intracraneanos rotos y no rotos incluye opciones quirúrgicas (clips vasculares) y endovasculares. Existen varios dispositivos y técnicas de manejo endovascular (coils, stents, diversores de flujo), cuyo conocimiento permite elegir e interpretar adecuadamente las técnicas de imágnes para el seguimiento no invasivo.

Mediante una revisión pictográfica del control de aneurismas intracraneanos tratados quirúrgicamente y vía endovascular, se muestran las diferencias en la capacidad de caracterización en el seguimiento con angio TC, angio RM sin contraste (con técnica de "tiempo de vuelo" o "TOF" por sus siglas en inglés), angio $R M$ con gadolinio endovenoso $(C+)$ y angiografía por sustracción digital (ASD). Adicionalmente se entregan recomendaciones para el seguimiento de aneurismas según modalidad y dispositivo de tratamiento. Palabras clave: aneurismas intracraneanos, coils, stent intracraneano, diversor de flujo, clips vasculares, seguimiento imagenológico.

Zerega M. Ensayo Pictórico: Control imagenológico de aneurismas tratados ¿Qué examen elegir? Rev Chil Radiol 2018; 24(2): 55-62.

Correspondencia: Sebastián Eduardo Bravo Grau. sebravo1@uc.cl

Trabajo enviado el 19 de julio de 2018. Aceptado para publicación el 18 de agosto de 2018.

\section{Introducción}

El tratamiento de aneurismas intracraneanos rotos y no rotos incluye opciones quirúrgicas (clips vasculares) y endovasculares. Existen múltiples dispositivos y técnicas de manejo endovascular (coils, stents, diversores de flujo). El seguimiento imagenológico es relevante por el riesgo de recanalización, crecimiento del aneurisma, rotura, re-rotura o aparición de nuevos aneurismas.
Existen diferentes modalidades de imagen para el seguimiento de aneurismas tratados, cuya elección dependerá del tipo de tratamiento empleado (tabla resumen). Mediante una revisión pictográfica del control de aneurismas intracraneanos tratados quirúrgicamente y vía endovascular, se muestran las diferencias en la capacidad de caracterización en el seguimiento con distintas técnicas: angiografía por tomografía computada (angio TC), angiografía por 
resonancia magnética (angio RM) sin contraste (en tiempo de vuelo o "TOF" por sus siglas en inglés), angio $\mathrm{RM}$ con gadolinio endovenoso $(\mathrm{C}+)$ y angiografía por sustracción digital (ASD). Adicionalmente se entregan recomendaciones para el seguimiento de aneurismas según modalidad de tratamiento (Tabla resumen).

Tabla Resumen. Modalidades imagenológicas según exactitud diagnostica para seguimiento de aneurismas tratados, según tipo de tratamiento.

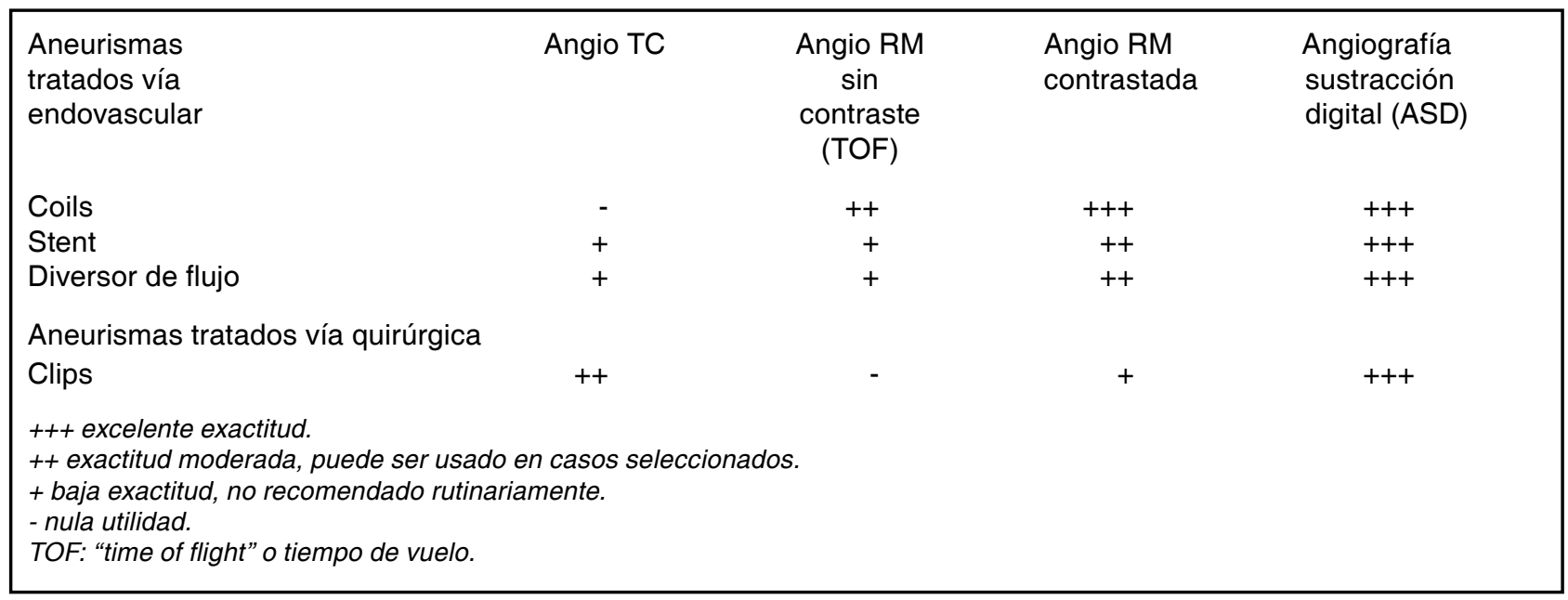

\section{Coils}

Los coils utilizados para embolización de aneurismas por vía endovascular son alambres que se enrollan en ovillos al ser liberados al interior del saco. Son fabricados con una aleación de platino para optimizar su visualización con técnica fluoroscópica durante su instalación ${ }^{1}$. La caracterización de los aneurismas tratados con coils en ASD se realiza mediante la clasificación de Raymond Roy modificada² (Figura 1).

Desafortunadamente el platino produce un acentuado artefacto de endurecimiento del haz de rayos $X$ en TC, lo que oscurece la información del parénquima y estructuras vasculares adyacentes.
Esto impide evaluar la presencia o ausencia de un aneurisma residual en el seguimiento con angio TC (Figura 2). Sin embargo, este material no tiene efecto ferromagnético, por lo que no altera en forma significativa el campo magnético local y no produce artefacto de desfase de señal en secuencias de TR corto como angio RM TOF o angio RM con contraste ${ }^{3}$. Con resonadores de 1,5 T el uso de medio de contraste permite detectar pequeños residuos que no generan señal de flujo en TOF (Figuras 3 y 4). En resonadores de $3 T$ la mejor relación señal/ruido permite detectar pequeños residuos sin uso de contraste ${ }^{4}$.

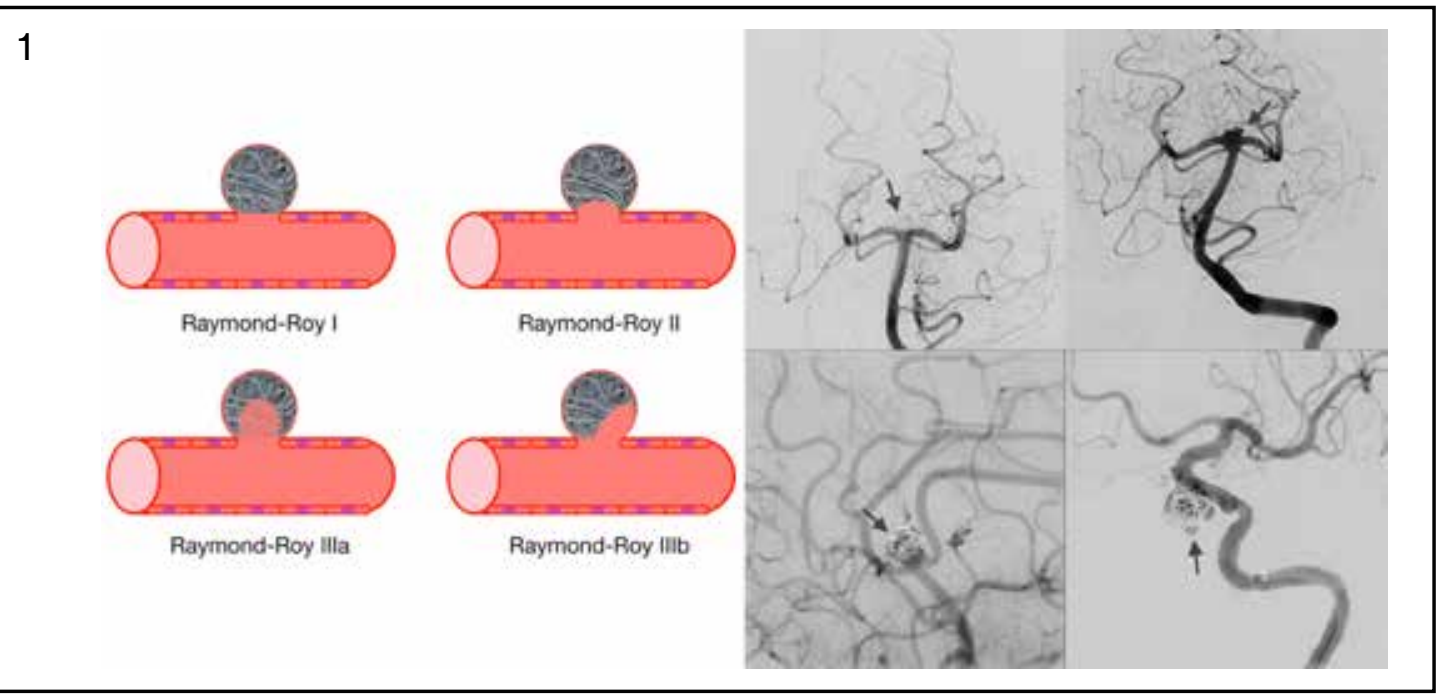

Figura 1. Coils - ASD - Clasificación de Raymond Roy modificada. Clase I obliteración total. Clase Il cuello residual. Clase IIla aneurisma residual con contraste en intersticios entre coils. Clase IIIb aneurisma residual con contraste entre pared del saco y coils. Las clases I y II se pueden agrupar en "cierre correcto". Clase Illa tiene mayor tendencia a evolucionar a I o II por lo que puede ser controlada. Clase Illb tiene indicación de re-tratamiento. 

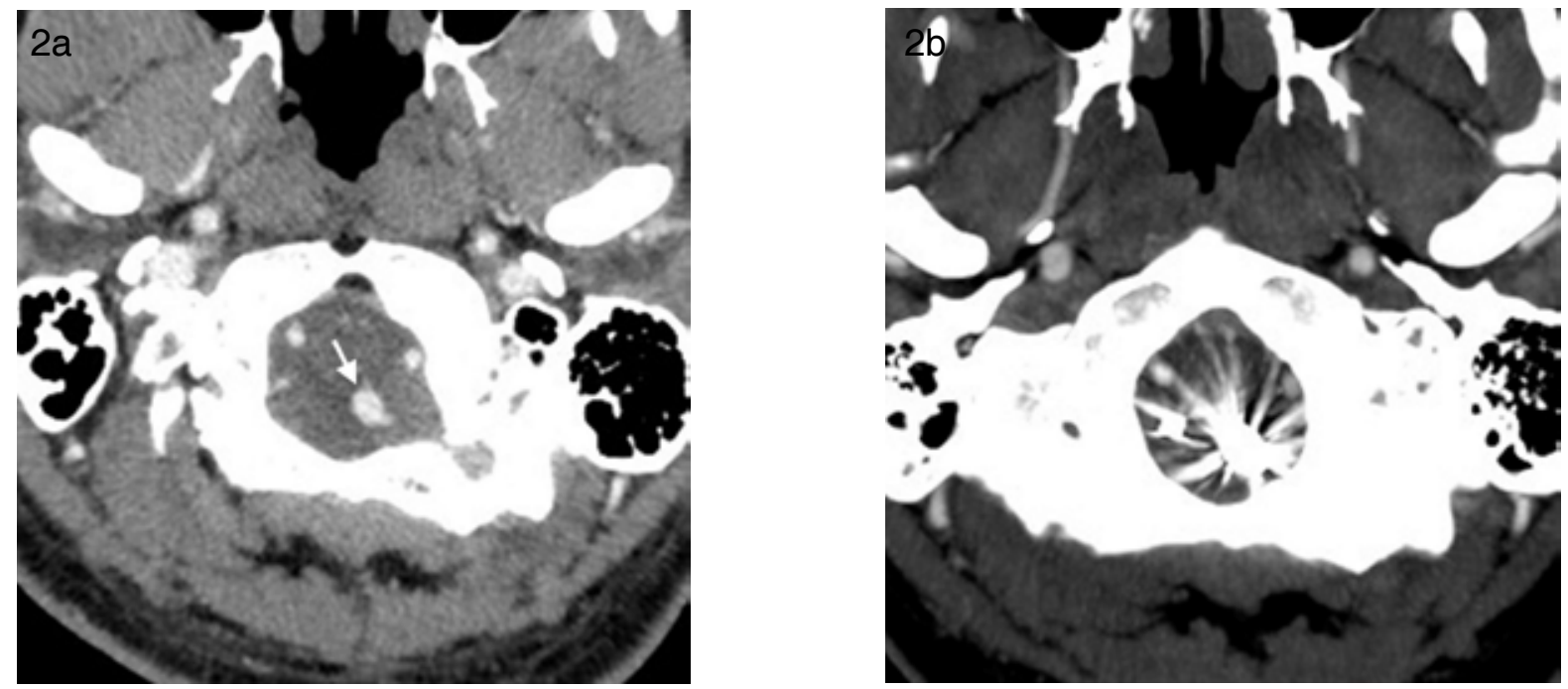

Figura 2. Coils - control con angio TC. La angio TC previa al tratamiento (a) muestra un aneurisma sacular de PICA izquierda (flecha). La angio TC de control (b) presenta marcado artefacto estrellado por endurecimiento del haz de rayos $X$, debido a la presencia de coils de platino. Esto impide la caracterización del saco aneurismático.

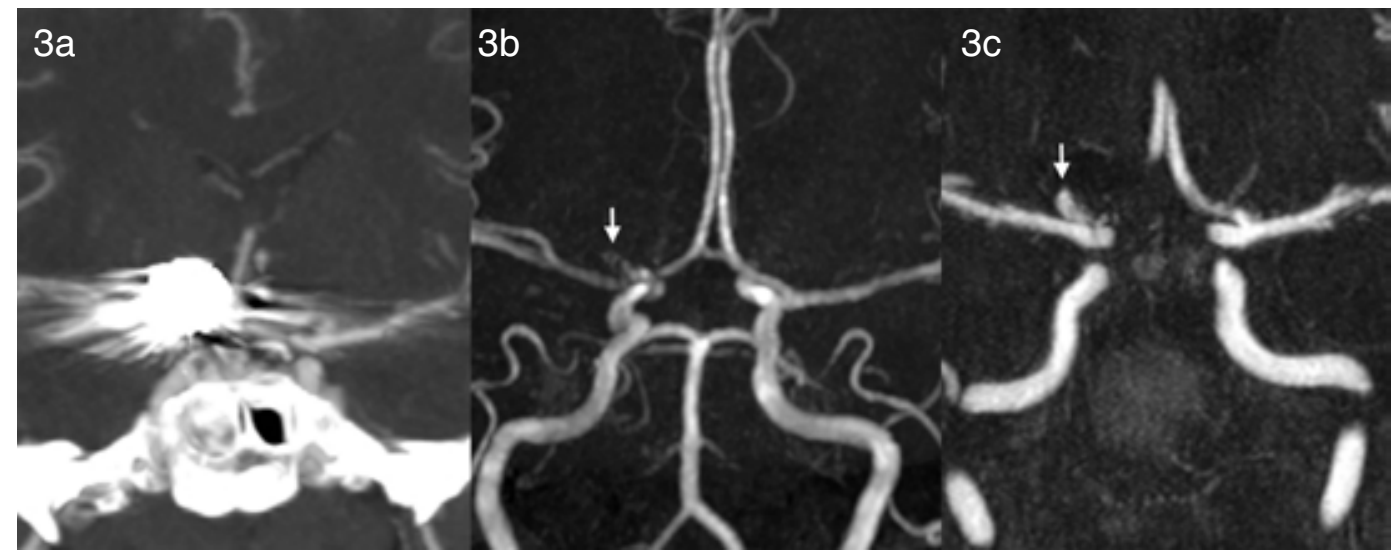

Figura 3. Coils - comparación entre angio TC, angio RM TOF y angio RM C+. Aneurisma carótido-oftálmico derecho tratado con coils. Angio TC (a), MIP 3D de angio RM TOF (b) y angio RM C+ (c). La angio TC no tiene utilidad por la presencia de artefactos (a). El aneurisma residual (flecha) se insinúa en TOF (b) pero es mejor demostrado en la angio $R M$ con contraste (c).
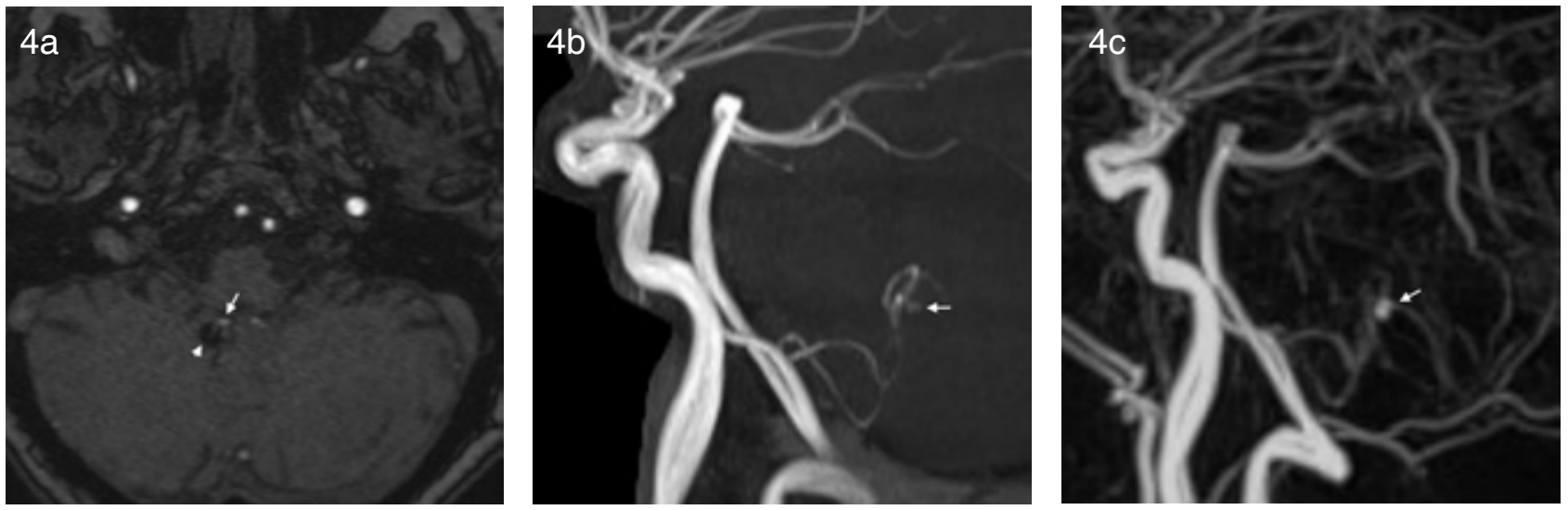

Figura 4. Coils - angio RM TOF y angio RM con medio de contraste $\left(C_{+}\right)$. Aneurisma de PICA derecha tratado con coils (punta de flecha en a). Similar al caso anterior el aneurisma residual de $5 \mathrm{~mm}$ se insinúa en TOF como escasa señal de flujo (flechas en a y b) pero es mejor demostrado en la angio RM C+ (flecha en $c$ ). 


\section{Clips}

La angio TC en muy superior a la angio RM para el seguimiento no invasivo del clipaje quirúrgico de aneurismas (Figura 5). Los clips de cobalto producen más artefactos en TC que los clips de titanio; éstos últimos son los más utilizados actualmente. Aunque la composición metálica de los clips quirúrgicos disminuye la penetración del haz de rayos $X$ y determina artefactos que degradan la imagen de tejidos adyacentes, el artefacto de susceptibilidad de campo magnético que determinan en RM produce ausencia de señal en un área mucho más extensa (Figuras 6 y 7 ). La angio TC puede mostrar y seguir pequeños remanentes aneurismáticos; demostrar permeabilidad, estenosis o vasoespasmo en el vaso parental; y vigilar la aparición de nuevos aneurismas post clipaje ${ }^{5}$. En presencia de múltiples clips, clips orientados paralelos al vaso parental o en el plano de adquisición, su rendimiento disminuye ${ }^{6}$.

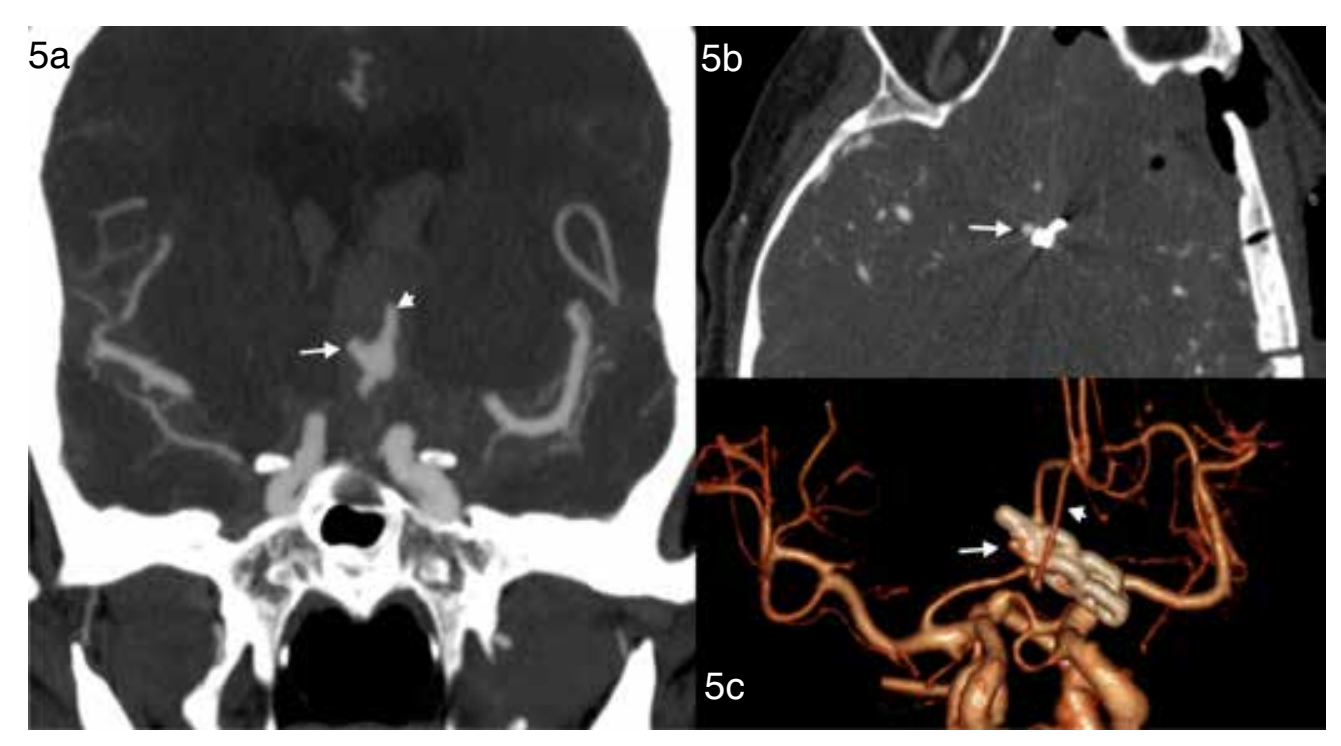

Figura 5. Clips - control con angio TC. Aneurisma sacular de cuello ancho de complejo anterior roto (a) con hemorragia subaracnoidea y hemoventrículo. El control de clipaje con angio TC muestra aneurisma residual con opacificación del mamelón de rotura (b: cortes axiales, c: MIP 3D). Además, se observa leve vasoespasmo difuso de A1 y A2 (puntas de flecha, comparar c y a). La angio TC es la técnica no invasiva de elección para diagnóstico de vasoespasmo.
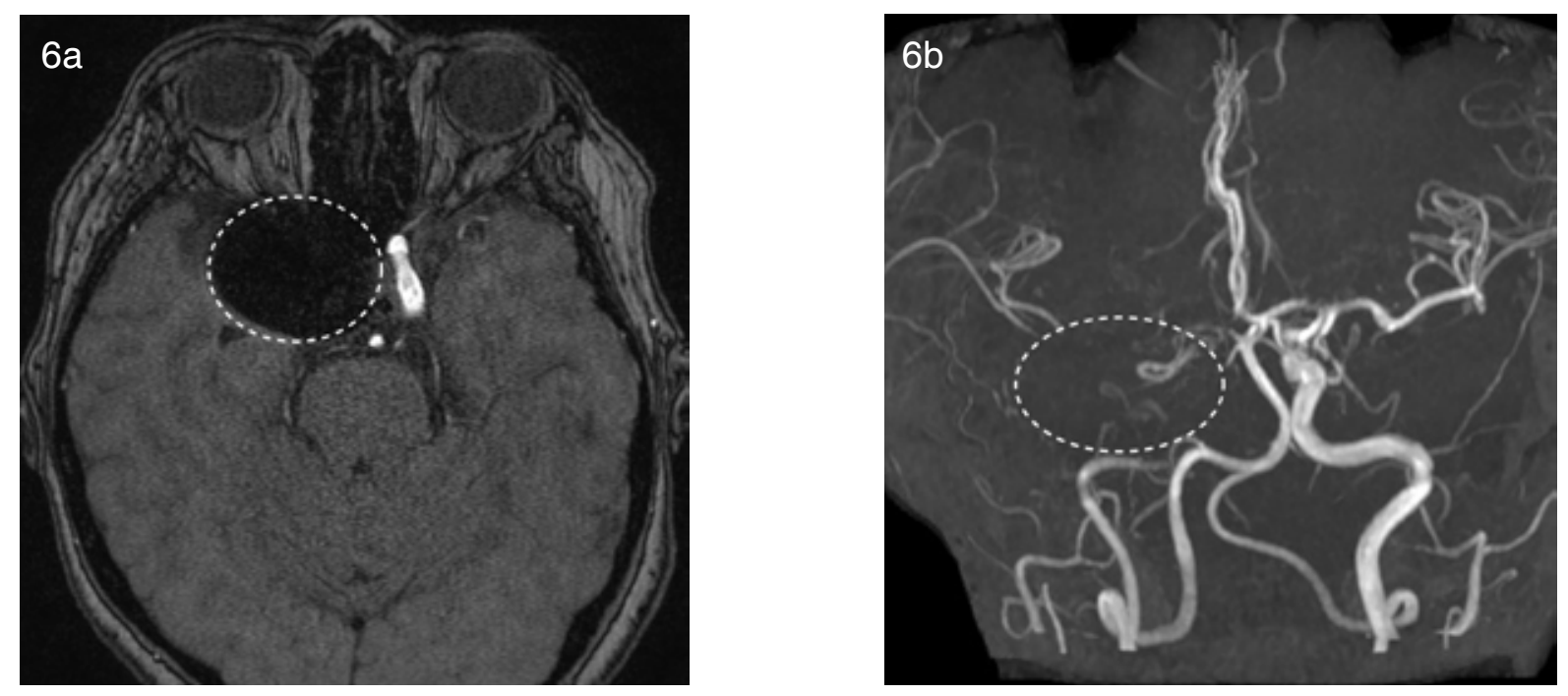

Figura 6. Clips - control con angio RM TOF. La angio-RM sin contraste con técnica de tiempo de vuelo en cortes axiales (a) y reformación MIP 3D (b) presenta ausencia de señal por artefacto de susceptibilidad (elipses en a y b) por presencia de clip vascular. A su vez, la ausencia de señal de flujo en ACl petrosa, cavernosa, supraclinoidea y en ACM proximal impide la evaluación del aneurisma tratado. 


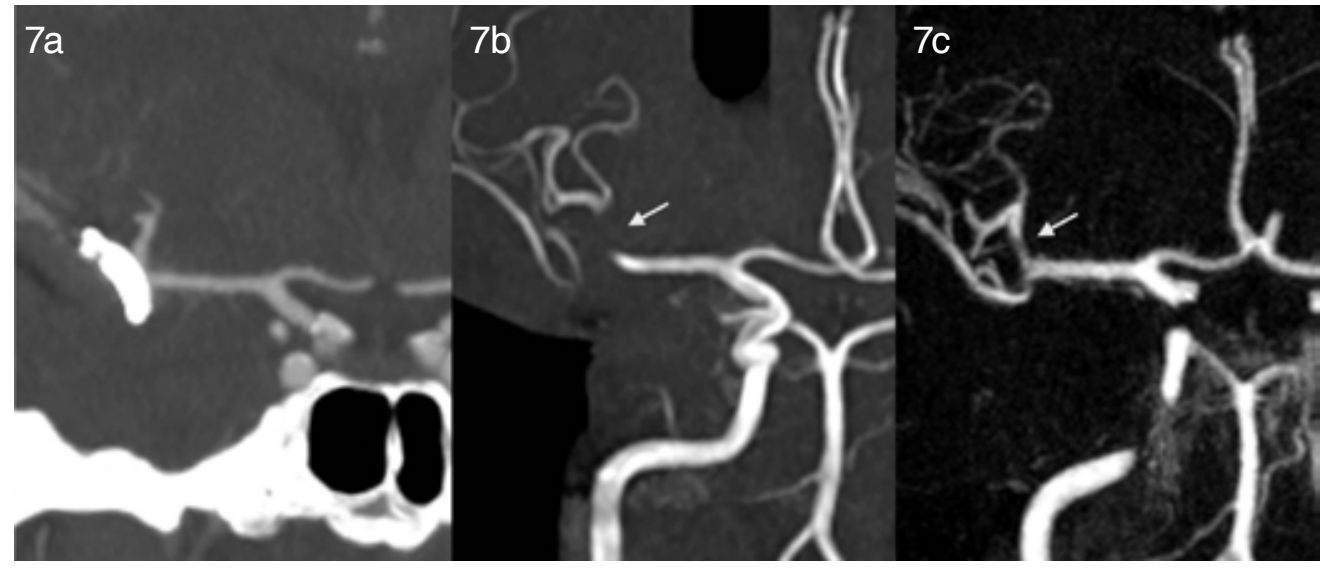

Figura 7. Clips - angio TC, angio RM TOF y angio RM C+. La angio TC (a) muestra un aneurisma de bifurcación de ACM derecha clipado, sin aneurisma residual y con adecuado flujo en M1 y M2. La angio RM TOF (b) muestra artefacto de susceptibilidad (flecha en b), lo que impide evaluar el vaso parental y el saco aneurismático. La angio RM C+ (c) muestra el vaso parental un poco mejor que angio RM TOF (flecha en c).

La clasificación de Sindou establece cinco categorías en cuanto a la presencia de aneurisma residual; los grados I y II presentan respectivamente opacificación menor a $50 \%$ y mayor a $50 \%$ del cuello del aneurisma; los grados III, IV y V adicionalmente al cuello remanente presentan opacificación de un lóbulo, de menos de $75 \%$ o más de $75 \%$ del saco aneurismático respectivamente7. El control durante la cirugía puede ser realizado con ASD, angiografía por fluorescencia con fluoresceína o indocianina ${ }^{8}$. Los remanentes con saco aneurismático (grados III, IV y V) son susceptibles a retratamiento, ya sea por vía quirúrgica o endovascular ${ }^{9}$.

\section{Stents}

Se usan stents para asistir el empleo de coils en aneurismas complejos (de cuello ancho, grandes o gigantes, fusiformes) ${ }^{10,11}$, para guiar el flujo sanguíneo en el vaso parental y reconstruir la pared arterial (Figura 8). Son fabricados con alambres de nitinol (aleación de níquel y titanio) y tienen marcadores radio-opacos metálicos en sus extremos. La incidencia de estenosis luminal en stents de pacientes tratados por aneurismas intracraneanos se ha estimado en $7,8 \%$ en seguimiento con $\mathrm{DSA}^{12}$, siendo esta asintomática. Existen pocos datos de la utilidad en el seguimiento de las técnicas angiográficas no invasivas ${ }^{13}$. Los stents producen artefacto de endurecimiento del haz de rayos $\mathrm{X}$, y disminución de señal luminal en angio RM TOF y $\mathrm{C}_{+}$, lo que limita la evaluación de estenosis luminal leve. Sin bien en nuestra experiencia las tres técnicas permiten establecer a groso modo la presencia de permeabilidad y descartar la presencia de estenosis de alto grado. La visualización del lumen en RM mejora con el uso de gadolinio (Figura 9).
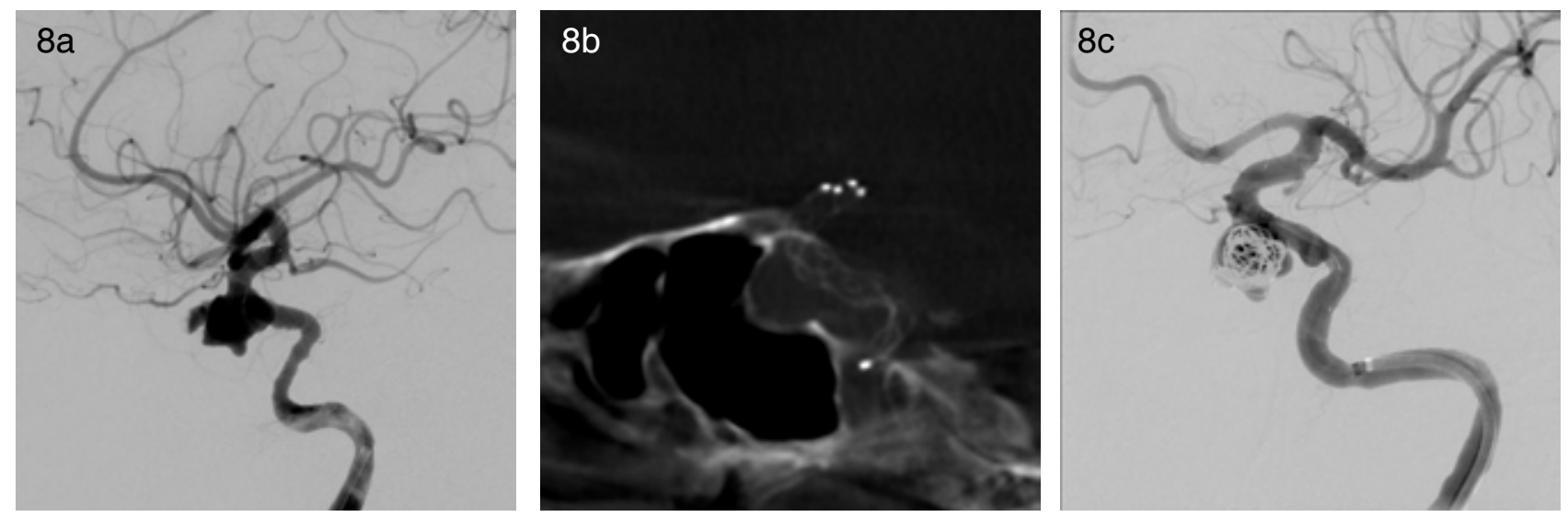

Figura 8. Stent - ASD. La ASD previa al tratamiento muestra un aneurisma de carótida interna cavernosa de orientación inferomedial. La TC "flat panel" MIP (b) del angiógrafo muestra un stent "solitaire". La ASD durante al tratamiento (c) muestra la masa de coils que se mantienen al interior del saco gracias a la presencia del stent. 

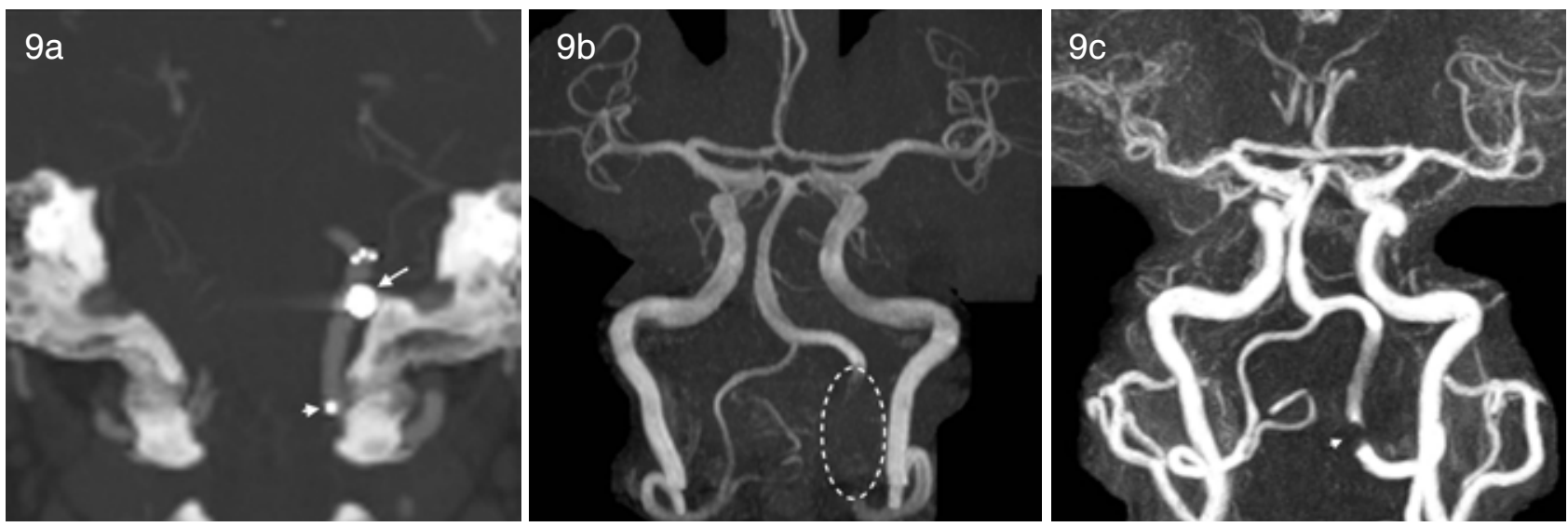

Figura 9. Stent + Coils. El control con angio TC muestra un stent con marcadores radiopacos proximal (punta de flecha) y distal con permeabilidad luminal. El saco ameurismático no es evaluable por presencia de coils (flecha blanca). En la angio RM TOF (b) el artefacto impide evaluar lumen y aneurisma residual (elipse). En la angio RM C+ (c) existe señal luminal "degradada" por susceptibilidad y mayor artefacto a nivel del marcador metálico proximal, pero no se observa aneurisma residual.

\section{Diversores de flujo}

Son dispositivos endoluminales tubulares cuyas paredes consisten en hebras metálicas de aleación cromo-cobalto o nitinol trenzadas. Son instalados de forma tal que cubran el cuello del aneurisma. Su objetivo es alterar el flujo de entrada y salida al aneurisma y de tal forma inducir trombosis del saco aneurismático ${ }^{14}$, sin ocluir ramas del vaso parental. En una revisión sistemática de su utilización en aneurismas no rotos se obtuvieron tasas de oclusión completa de $76 \%$, de morbilidad de $5 \%$ y de mortalidad de $4 \%^{15}$. La tasa de infartos en territorio de arterias perforantes fue de $3 \%$, significativamente menor en territorios de circulación anterior vs posterior. Otra revisión sistemática en aneurismas rotos determinó mayores tasas de oclusión $(90 \%$, con resultado clínico favorable en $81 \%$ ) a expensas de una mayor tasa de morbilidad $(12 \%)^{16}$. La tasa de rerotura fue de $5 \%, 67 \%$ de estas en aneurismas mayors de $2 \mathrm{~cm}$. A pesar de no ser la primera línea de tratamiento, la utilización de diversores de flujo es una alternativa válida en aneurismas de tratamiento difícil por técnicas convencionales.

Los diversores de flujo producen artefacto de endurecimiento del haz de rayos $X$, y disminución de señal luminal en angio RM TOF y $\mathrm{C}+$. Sin embargo, esto no limita la evaluación de un saco aneurismático residual o remanente (Figuras 10, 11 y 12). Al igual

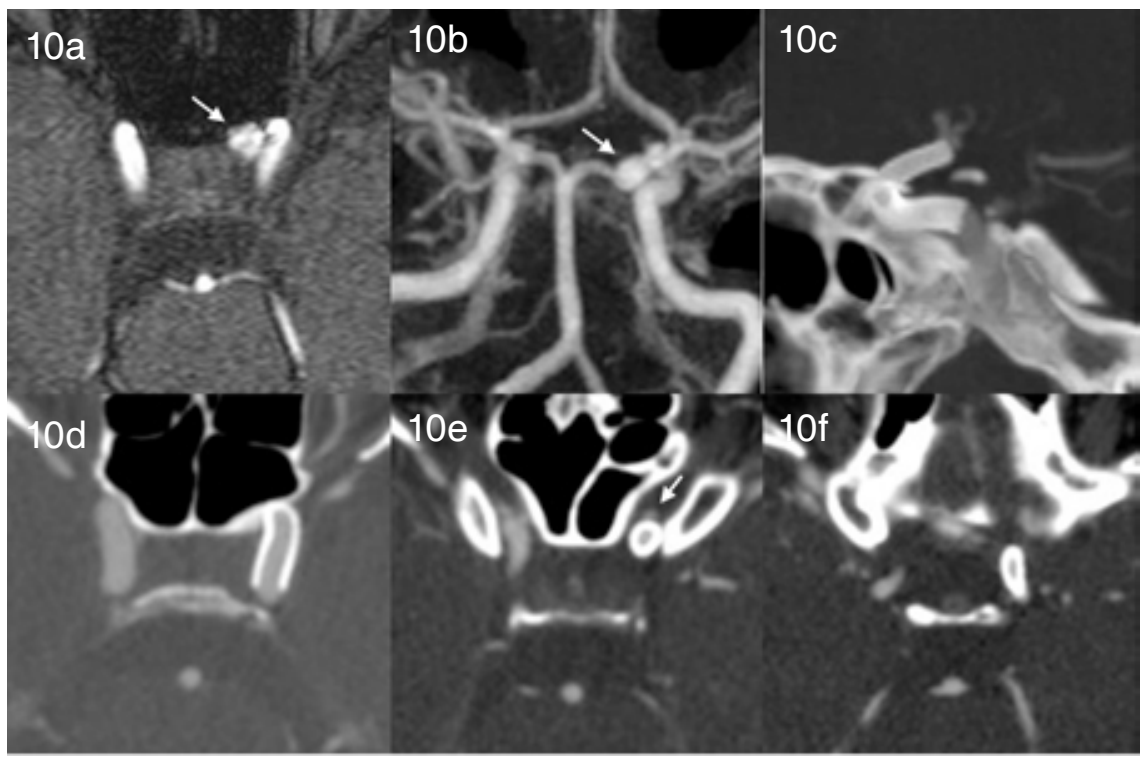

Figura 10. Diversor de flujo - Control con Angio TC. La angio RM TOF axial (a) y MIP 3D (b) muestran un aneurisma de sifón carotídeo izquierdo de orientación medial (flecha). El control inmediatamente terminado el procedimiento con TC "flat panel" del angiógrafo exhibe un diversor de flujo bien posicionado (c). El control posterior con angio TC presenta permeabilidad luminal (d-f) y de la arteria oftálmica (flecha en e). 

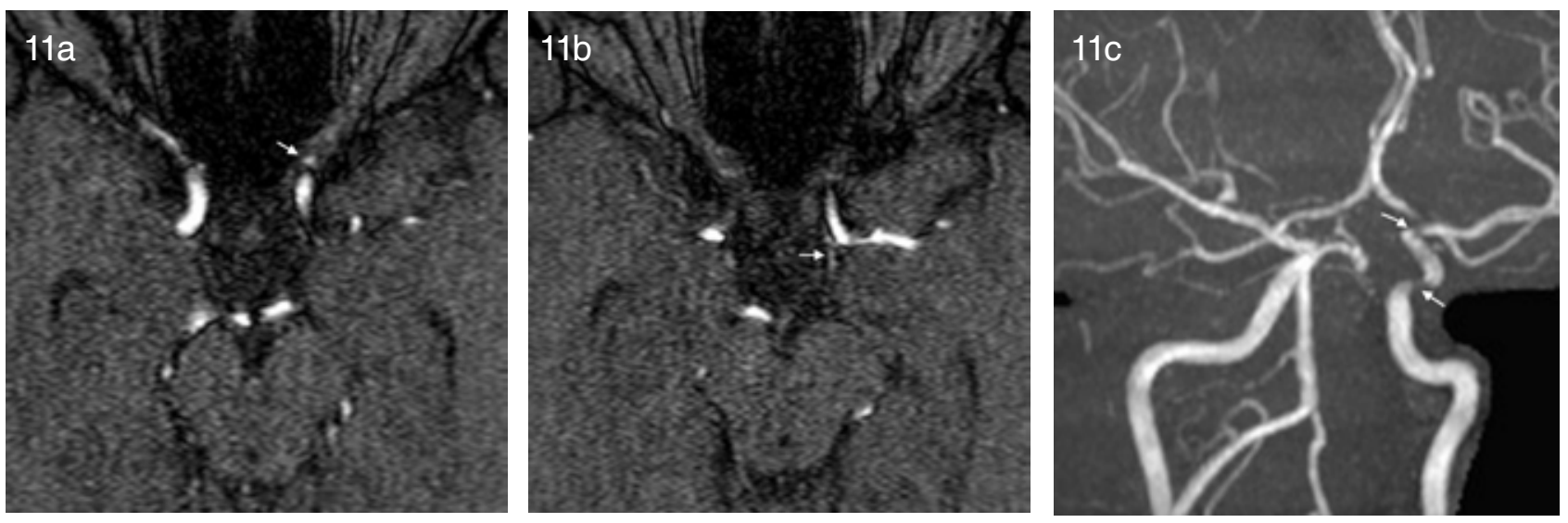

Figura 11. Diversor de flujo - Angio RM TOF. El mismo paciente que en figura anterior. Existe adecuada señal luminal (a-c) en arterias oftálmica (flecha en a) y comunicante posterior izquierdas (flecha en b), sin evidencia de aneurisma residual. La proyección MIP 3D oblicua (c) demuestra artefacto por susceptibilidad magnética más acentuado en los extremos del diversor (flechas en $c$ ).
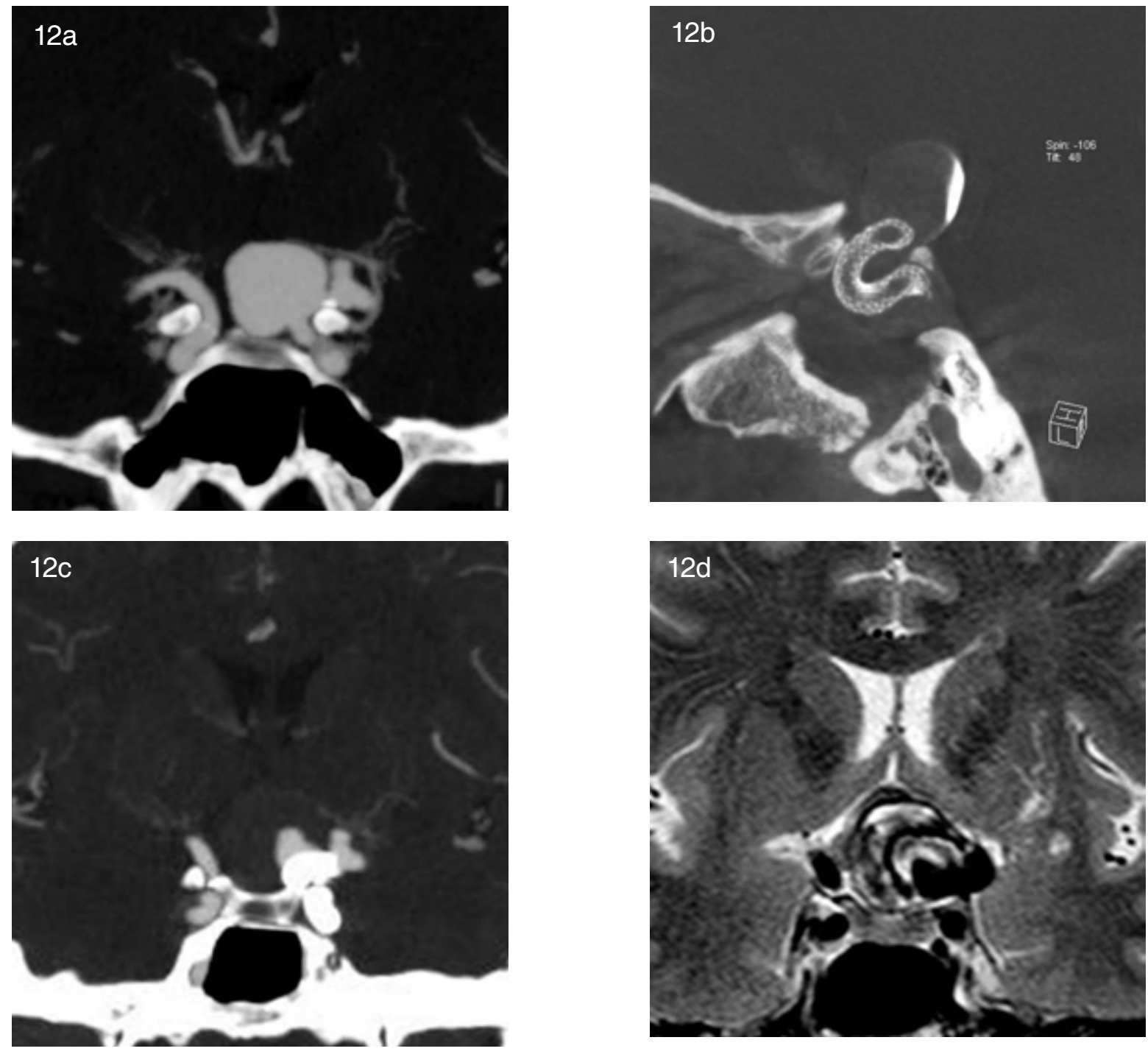

Figura 12. Diversor de flujo - angio TC - ASD (3D) - RM. Aneurisma de carótida interna supraclinoidea que compromete el origen de la arteria cerebral media izquierda en MIP coronal de angio TC (a). La TC "flat panel" MIP (b) muestra un diversor de flujo con acumulación de contraste en posición declive, lo que traduce estasia de flujo. La angio TC (c) presenta disminución de tamaño del lumen y la RM (d) trombosis del saco aneurismático. 
que con los stents es posible determinar presencia de permeabilidad y descartar la presencia de estenosis de alto grado. La visualización del lumen en RM mejora con el uso de gadolinio.

\section{Discusión}

La ASD es el "gold standard" para el diagnóstico de aneurismas. Sin embargo, es invasiva y tiene morbilidad permanente (aunque es baja 0,5\%). Mas aun, su uso reiterado en el seguimiento podría resultar en aumento acumulativo de morbilidad a largo plazo, lo que podría disminuir el beneficio del tratamiento'.

El seguimiento de aneurismas tratados por vía endovascular debe partir con la valoración del resultado con ASD finalizado el procedimiento. En el seguimiento alejado de Coils se debería usar angio $\mathrm{RM} \mathrm{C}+$ que tiene mayor rendimiento que TOF, y similar rendimiento que ASD para toma de decisiones de manejo. El artefacto producido por el platino en TC impide evaluar el saco aneurismático con angio TC (Figuras 2 y 3 ). Aneurismas clipados deberían seguirse con angio TC, eventualmente con angio RM $\mathrm{C}+$ pero no con angio RM TOF, esta última limitada por artefacto de susceptibilidad (Figura 6). Stents y diversores de flujo pueden ser seguidos con ambas técnicas. Si están acompañados de coils se debe

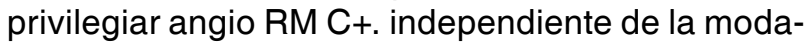
lidad de tratamiento, la angio TC es la técnica no invasiva de elección para monitorizar la aparición de vasoespasmo en el contexto de HSA aneurismática, y siempre se debe usar como base de comparación el estudio de ingreso.

\section{Referencias}

1. Wallace RC, Karis JP, Partovi S, Fiorella D. Noninvasive imaging of treated cerebral aneurysms, part I: MR angiographic follow-up of coiled aneurysms. Am J Neuroradiol. 2007; 28(6): 1001-1008.

2. Mascitelli JR, Moyle H, Oermann EK, Polykarpou MF, Patel AA, Doshi AH, et al. An update to the RaymondRoy Occlusion Classification of intracranial aneurysms treated with coil embolization. J Neurointerv Surg. 2015; 7(7): 496-502.

3. Pjontek R, Önenköprülü B, Scholz B, Kyriakou Y, Schubert GA, Nikoubashman $O$, et al. Metal artifact reduction for flat panel detector intravenous CT angiography in patients with intracranial metallic implants after endovascular and surgical treatment. J Neurointerv Surg. 2016; 8(8): 824-
829.

4. Anzalone N, Scomazzoni F, Cirillo M, Righi C, Simionato F, Cadioli M, et al. Follow-up of coiled cerebral aneurysms at 3T: Comparison of 3D time-of-flight MR angiography and contrast-enhanced MR angiography. Am J Neuroradiol. 2008; 29(8): 1530-1536.

5. Wallace RC, Karis JP, Partovi S, Fiorella D. Noninvasive imaging of treated cerebral aneurysms, part II: CT angiographic follow-up of surgically clipped aneurysms. Am J Neuroradiol. 2007; 28(7): 1207-1212.

6. Masaryk AM, Frayne R, Unal O, Rappe AH, Strother CM. Utility of CT angiography and MR angiography for the follow-up of experimental aneurysms treated with stents or Guglielmi detachable coils. Am J Neuroradiol. 2000; 21(8): 1523-1531.

7. Sindou M, Acevedo JC, Turjman F. Aneurysmal remnants after microsurgical clipping: Classification and results from a prospective angiographic study (in a consecutive series of 305 operated intracranial aneurysms). Acta Neurochir (Wien). 1998; 140(11): 1153-1159.

8. Kakucs C, Florian IA, Ungureanu G, Florian IS. Fluorescein Angiography in Intracranial Aneurysm Surgery: A Helpful Method to Evaluate the Security of Clipping and Observe Blood Flow. World Neurosurg. 2017; 105: 406-411.

9. Pierot L, Wakhloo AK. Endovascular treatment of intracranial aneurysms: Current status. Stroke. 2013; 44(7): 2046-2054.

10. Juvela S, Poussa K, Lehto H, Porras M. Natural history of unruptured intracranial aneurysms: A long-term follow-up study. Stroke. 2013; 44(9): 2414-2421.

11. Yoon KW, Kim YJ. In-stent stenosis of stent assisted endovascular treatment on intracranial complex aneurysms. J Korean Neurosurg Soc. 2010; 48(6): 485-489.

12. Soize S, Gawlitza M, Raoult H, Pierot L. Imaging Follow-Up of Intracranial Aneurysms Treated by Endovascular Means: Why, When, and How? Stroke. 2016; 47(5): 1407-1412.

13. Kallmes DF, Ding YH, Dai D, Kadirvel R, Lewis DA, Cloft HJ. A second-generation, endoluminal, flow-disrupting device for treatment of saccular aneurysms. Am J Neuroradiol. 2009; 30(6): 11531-1158.

14. Brinjikji W, Murad MH, Lanzino G, Cloft HJ, Kallmes DF. Endovascular treatment of intracranial aneurysms with flow diverters: A meta-analysis. Stroke. 2013: 44; 442-447.

15. Madaelil TP, Moran CJ, Cross DT 3rd, Kansagra AP, T.P. $M$, C.J. $M$, et al. Flow diversion in ruptured intracranial aneurysms: A meta-analysis. Am J Neuroradiol. 2017 Mar; 38(3): 590-595.

16. X F. Cagnazzo, X D.T. di Carlo, X M. Cappucci, X P.-H. Lefevre, X V. Costalat XPP. Acutely Ruptured Intracranial Aneurysms Treated with Flow-Diverter Stents : A Systematic Review and Meta-Analysis. AJNR Am J Neuroradiol. 2018; $1-7$. 